\title{
Distribution Analysis of Hydroxyl Groups in Polymers by Derivatization-Electron Probe X-ray Microanalysis
}

\author{
Motoyasu Sugiura,* Takuya Mitsuoka,* Atsushi Murase,* and Kazumasa Ueda** \\ * Materials Analysis \& Evaluation Div., Toyota Central R \& D Labs. Inc., Nagakute, Aichi 480-1192, Japan \\ **Department of Chemistry and Chemical Engineering, Kanazawa University, \\ Kodatsuno, Kanazawa 920-8667, Japan
}

\begin{abstract}
An analytical method, referred to as "derivatization-electron probe X-ray microanalysis (XMA)", has been developed to determine the distribution of small amounts of hydroxyl groups formed by the oxidation and the hydrolysis of polymers. A suitable condition for a derivatization reaction with hydroxyl groups in polymers was investigated. It was found that hydroxyl groups in polymers were derivatized selectively using gas-phase esterification with trichloroacetic anhydride (TCAA). The most suitable amount of TCAA in a 50-ml vial was $50 \mu \mathrm{l}$. After setting a sample in the vial without directly contacting the reagent, by heating the reagent and the sample at $60^{\circ} \mathrm{C}$ for $90 \mathrm{~min}$, the highest reaction yield and selectivity were obtained. By derivatization-XMA using this reaction condition, a distribution measurement of small amounts of hydroxyl groups in the polymer became feasible. Actual applications to a depth analysis of degraded polyethylene and polyester proved that the present method is useful for the characterization of polymers and for the study of polymer degradation.
\end{abstract}

(Received June 30, 2000; Accepted October 3, 2000)

Polymer materials degrade with the operation of environment factors, such as light and heat, and lose their function as polymers. Therefore, it is important to study the degradation mechanism of polymers and to use an evaluation test that reflects their environment. Also, in the degradation evaluation, a chemical evaluation in addition to a physical evaluation is necessary. In the chemical evaluation, changes in the composition and the number of functional groups that occur with the degradation are examined in the surface and a section of the polymer material.

Since infrared absorption spectroscopy (IR) provides most valuable information about the chemical structure, and can be used for micro measurements (micro-IR), the use of IR for a distribution analysis of the compositions and the functional groups is increasing. ${ }^{1-3}$ Especially, in the depth analysis, a depth resolution of $1 \mu \mathrm{m}$ has been achieved, and IR has been used for a degradation evaluation of polymer materials and so on. ${ }^{4}$ However, a change of less than several percent of the components can not often be measured by IR. Therefore, a long evaluation test period is necessary to study the degradation mechanism and to evaluate the degradation degree.

"Derivatization-electron probe X-ray microanalysis (XMA)" was developed to measure the distribution of small amounts of functional groups generated in a degraded polymer with a lateral resolution of $\mu \mathrm{m}$ order using generally available equipment. $^{5-7}$ In this study, the optimal conditions for a derivatization reaction with hydroxyl groups in polymers were investigated. Also, the utility of this method in studying the degradation mechanism and in evaluating the degradation degree was confirmed by applying this method to a distribution analysis of hydroxyl groups in degraded polyethylene and polyester.

\section{Experimental}

\section{Choice of derivatization reagent}

Various derivatization reactions of the hydroxyl group have been examined for the purpose of labeling in X-ray photoelectron spectroscopy (XPS). ${ }^{8-10} \quad$ Representative derivatization reagents are trifluoroacetic anhydride $\left(\left(\mathrm{CF}_{3} \mathrm{CO}\right)_{2} \mathrm{O}\right)$, tribromoacetic acid $\left(\mathrm{CBr}_{3} \mathrm{COOH}\right)$ and 4chlorophenyl isocyanate $\left(\mathrm{ClC}_{6} \mathrm{H}_{4} \mathrm{NCO}\right)$. A reaction that uses trifluoroacetic anhydride is a gas-phase reaction and the reactions that use other reagents are liquid-phase reactions. Thus, an esterification reaction that used trifluroacetic anhydride as the derivatization reagent was chosen. Because this reaction is a gas-phase reaction, the reactivity is high and damage to a sample is small. However, trifluoroacetic anhydride has an F element with high sensitivity to XPS, but low sensitivity to XMA. Therefore, using trichloroacetic anhydride (TCAA), which replaces the $\mathrm{F}$ element with the $\mathrm{Cl}$ element, as a new derivatization reagent for the hydroxyl group, the optimal derivatization condition was investigated. Moreover, solid trichloroacetic acid is formed at the room temperature in TCAA and hydroxyl group reactions. Therefore, the condition necessary to remove this compound must also be investigated.

\section{Sample and reagent}

As polymers with hydroxyl groups in their structure, ethylenevinyl alcohol copolymer (EVA, Kurare Co., Ltd), which has hydroxyl groups in the main chain and poly-2-hydroxylethyl methacrylate (HEMA, General Science Co., Ltd), which has hydroxyl groups in the side chain, were used. A film of EVA approximately $500 \mu \mathrm{m}$ thick was prepared by the hot-press 


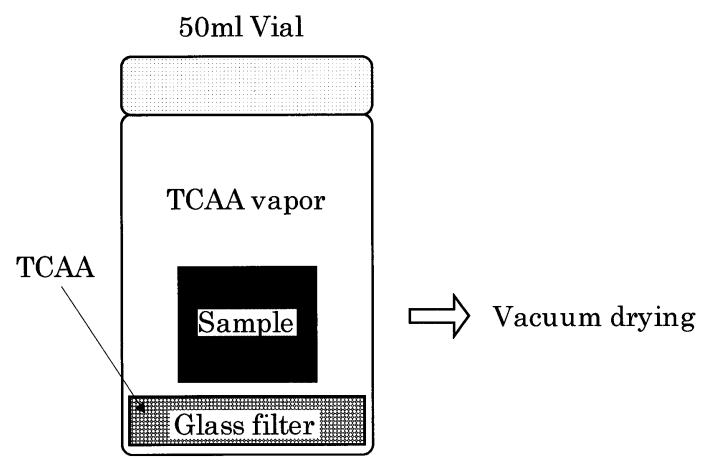

Fig. 1 Schematic illustration of a reaction instrument for the derivatization of hydroxyl groups in polymers.

method and a film of HEMA approximately $200 \mu \mathrm{m}$ thick was prepared by casting an ethanol solution on a glass plate.

Plates or films of the following polymers that had functional groups, except a hydroxyl group, were prepared to confirm the selectivity of the reaction: ethylene-butadiene rubber (EBR, General Science Co., Ltd.), which has vinyl groups; ethylenecarbon monoxide copolymer (E-CO, Japan Yunika Co., Ltd), which has carbonyl groups; polyacrylic acid (PAA, Sumitomo Chemistry Co., Ltd), which has carboxyl groups; polymetyl methacrylate (PMMA, General Science Co., Ltd), which has ester groups; Novolak-type epoxy resin (N-epoxy, General Science Co., Ltd), which has epoxy groups; and Nylon-6 (Ny-6, Toray Co., Ltd), which has amide groups.

As the derivatization reagent, trichloroacetic anhydride (TCAA, Aldrich Co., Ltd) was used.

\section{Derivatization of hydroxyl group}

An outline of the derivatization is shown in Fig. 1. The sample was put on a glass filter in the vial and the reaction reagent was poured into the glass filter without directly contacting the sample. The vial was then heated in an oven. After the reaction, the sample was vacuum dried so as to exclude any reaction reagent that had penetrated the sample and also the by-product, trichloroacetic acid.

\section{Measurement condition}

A line analysis by XMA was performed using a Shimadzu EPM-810Q with a beam diameter, acceleration voltage, sample electric current and scan speed of $1 \mu \mathrm{m}, 20 \mathrm{kV}, 20 \mathrm{nA}$ and 50 $\mu \mathrm{m} / \mathrm{min}$, respectively. The thickness of the gold coating was 5 $\mathrm{nm}$ (coating time: $1 \mathrm{~min}$ ) and as the detection $\mathrm{X}$-rays, $\mathrm{K}_{\alpha}$-rays $(0.4728 \mathrm{~nm})$ were used for the $\mathrm{Cl}$ element. The concentration (C) of the elements was found from formula (1) by the ZAF revision method generally used in XMA,

$$
C=G(k) \cdot k \text {. }
$$

Here, $G(k)$ is a revision coefficient and $k$ is the X-ray intensity of the sample/X-ray intensity of the standard substance. $\mathrm{KCl}$ was used as the standard substance.

In a line analysis by micro-IR, a JEOL JIR-100 type Fourier transform infrared spectrometer with an MAU110 type microIR unit was used, with a slit size and a step sending width of 20 $\mu \mathrm{m} \times 50 \mu \mathrm{m}$ and $20 \mu \mathrm{m}$, respectively.

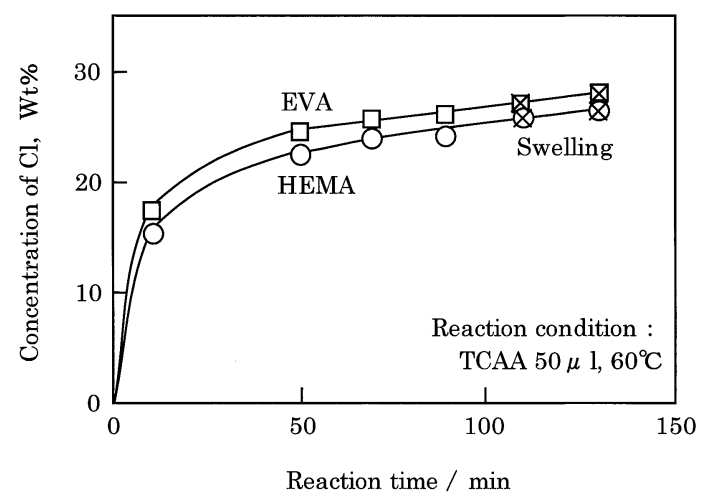

Fig. 2 Relationship between the reaction time and the $\mathrm{Cl}$ concentration obtained by XMA after derivatization.

\section{Results and Discussion}

Derivatization condition of the hydroxyl group

The optimal quantity of TCAA as the derivatization reagent was investigated by fixing the temperature and the time of the derivatization reaction at $60^{\circ} \mathrm{C}$ and $90 \mathrm{~min}$, respectively. EVA and HEMA as the polymers with hydroxyl groups were used. The relationship between the TCAA quantity in a 50-ml vial and the $\mathrm{Cl}$ concentration ( $\propto$ reaction yield) obtained by an XMA measurement after the reaction was examined. The TCAA quantity was changed with $30,50,100 \mu \mathrm{l}$. As a result, for both EVA and HEMA, the reaction yield increased with increasing TCAA quantity between $30 \mu \mathrm{l}$ and $100 \mu \mathrm{l}$. However, at $100 \mu \mathrm{l}$ TCAA, the sample completed swelling. Therefore, the optimal TCAA quantity was fixed at $50 \mu \mathrm{l}$ in the $50-\mathrm{ml}$ vial, because the sample showed no change at $50 \mu$ l.

Next, the optimal reaction temperature was investigated by fixing the reagent quantity and the time of the derivatization reaction at $50 \mu \mathrm{l}$ in the $50-\mathrm{ml}$ vial and $90 \mathrm{~min}$, respectively. The relationship between the reaction temperature and the $\mathrm{Cl}$ concentration obtained by an XMA measurement after the reaction was examined. The reaction temperature was changed to $50,60,70^{\circ} \mathrm{C}$. As a result, for both EVA and HEMA, the reaction yield increased with increasing the reaction temperature between $50^{\circ} \mathrm{C}$ and $70^{\circ} \mathrm{C}$. However, at $70^{\circ} \mathrm{C}$, the sample completed swelling. Therefore, the reaction temperature was fixed at $60^{\circ} \mathrm{C}$, because the sample showed no change there.

The optimal reaction time was investigated by fixing the reagent quantity and the temperature of the derivatization reaction at $50 \mu \mathrm{l}$ in the $50-\mathrm{ml}$ vial and $60^{\circ} \mathrm{C}$, respectively. The relationship between the reaction time and the $\mathrm{Cl}$ concentration obtained by an XMA measurement after the reaction is shown in Fig. 2. As a result, for both EVA and HEMA, the reaction yield increased with increasing reaction time. However, at 110 min, the sample completed swelling. Therefore, the reaction time was fixed at $90 \mathrm{~min}$, because the sample showed no change there.

The vacuum drying time to remove any non-reacting reagent and the by-product from the sample was investigated. As the sample to examine the removal time of non-reacting reagent, PMMA, which has no hydroxyl group, was used. As samples to examine the removal time of the by-product, EVA and HEMA, which have hydroxyl groups, were used. As the derivatization conditions, the quantity of TCAA, the reaction temperature and the reaction time were fixed at $50 \mu \mathrm{l}$ in a $50-\mathrm{ml}$ vial, $60^{\circ} \mathrm{C}$ and $90 \mathrm{~min}$, respectively. The vacuum drying temperature was 


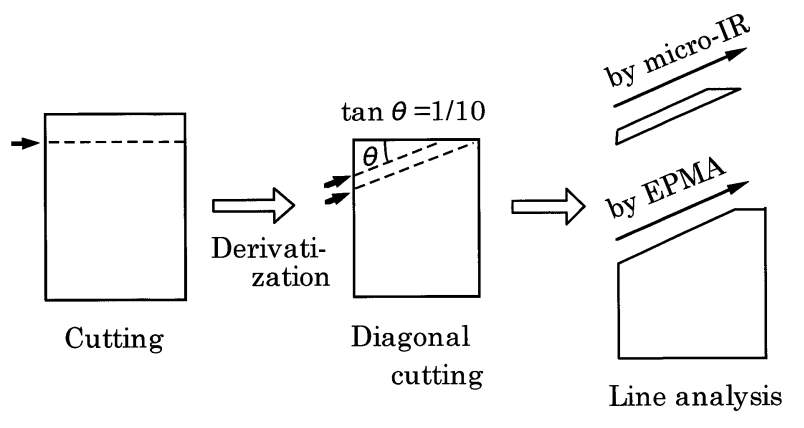

Fig. 3 Schematic drawing of the measurement of the derivatization reaction depth.
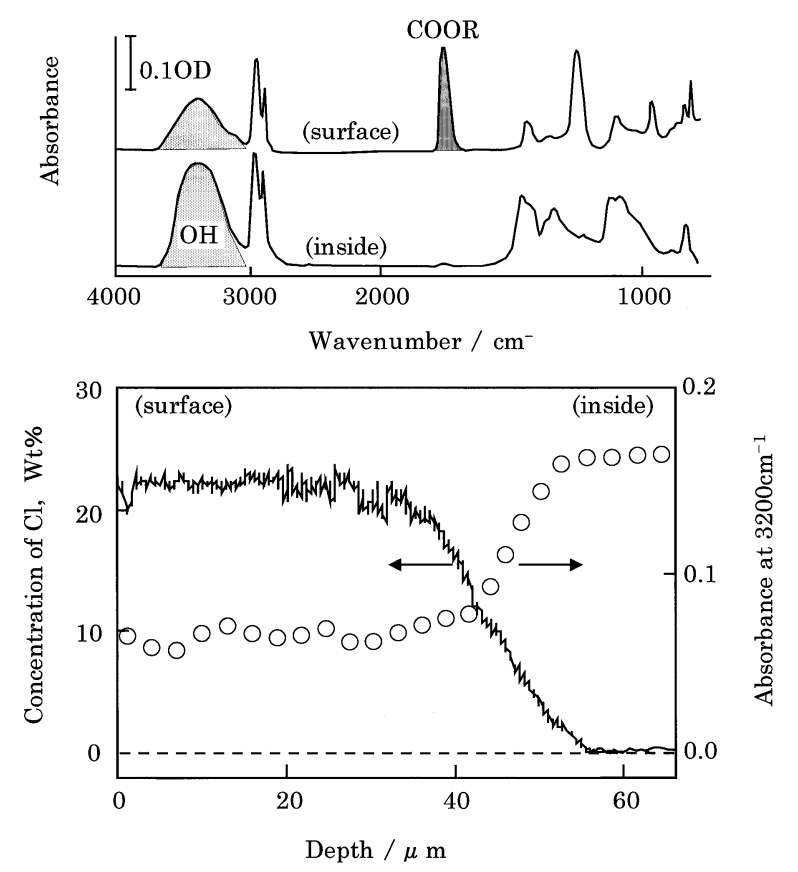

Fig. 4 Depth profiles of hydroxyl groups in derivatized ethylenevinylalcohol copolymer (EVA) for measuring the reaction depth (lower) and IR spectra of derivatized EVA (upper).

fixed at $60^{\circ} \mathrm{C}$, the same as the reaction temperature. The relationship between the vacuum drying time and the remaining $\mathrm{Cl}$ concentration was investigated. As a result, in PMMA, which has no hydroxyl group, the $\mathrm{Cl}$ concentration was high in the early stages of drying, and attenuated to approximately zero after $30 \mathrm{~h}$. This result means that the reaction reagent which dose not react to PMMA can be approximately removed by vacuum drying at $60^{\circ} \mathrm{C}$ for $30 \mathrm{~h}$. On the other hand, for EVA and HEMA, which have hydroxyl groups, the $\mathrm{Cl}$ concentrations decreased up to $5 \mathrm{~h}$ drying, and there was no subsequent change. This result means that the non-reacting reagent and the by-product were removed. Therefore, the vacuum drying temperature and time were fixed at $60^{\circ} \mathrm{C}$ for $30 \mathrm{~h}$.

\section{Performances of derivatization-XMA}

The reaction depth was examined in the way shown in Fig. 3. After cutting a sample in a microtome, the cutting surface was derivatized under the optimum conditions and the reaction surface was cut diagonally. The cutting surface was analyzed by a line analysis in XMA, and the film (1 $\mu \mathrm{m}$ thickness) obtained by cutting was analyzed by a line analysis in micro-IR.
Table $1 \mathrm{Cl}$ concentration obtained by XMA after derivatization in polymers having various functional groups

\begin{tabular}{llc}
\hline Polymer & Functional group & Conc. of $\mathrm{Cl}(\mathrm{wt} \%)$ \\
\hline EVA & hydroxyl group & 24 \\
HEMA & hydroxyl group & 21 \\
EBR & vinyl group & $<0.06$ \\
E-CO & carbonyl group & $<0.06$ \\
PAA & carboxyl group & $<0.06$ \\
PMMA & ester group & 1.8 \\
N-Epoxy & epoxy group & 12 \\
Ny-6 & amide group & 22 \\
\hline
\end{tabular}

The distribution of $\mathrm{Cl}$ elements obtained by XMA and the distribution of hydroxyl groups obtained by micro-IR are shown in the lower section of Fig. 4. The depth from the reaction surface is shown by the horizontal axis, the $\mathrm{Cl}$ concentration measured by XMA is shown by the left vertical axis, and the absorbance at $3200 \mathrm{~cm}^{-1}(v \mathrm{OH})$ measured by micro-IR is shown by the right vertical axis. From this result, that the reaction reached $50 \mu \mathrm{m}$ depth was confirmed, because the depth with decreasing $\mathrm{Cl}$ element and the depth with increasing hydroxyl group agreed. This depth is deeper than the detection depth $(10 \mu \mathrm{m})^{5}$ of XMA in polymer.

Also, the IR spectra of the surface and the inside are shown in the upper section of Fig. 4. From these spectra, that the derivatization reaction evidently advanced was proved, because the absorbance of hydroxyl group $\left(3200 \mathrm{~cm}^{-1}\right)$ in the surface (the reaction part) was decreased more than that inside (the nonreaction part), and the absorption of ester $\left(1740 \mathrm{~cm}^{-1}\right)$ was increased instead. Moreover, the reaction yield was about 50\%, because the absorbance of the non-reaction part (the inside) was 0.18 and the absorbance of the reaction part (the surface) was 0.08. This result may be because the molecular weight of the derivatization regent was too big, or because the half of the hydroxyl groups in EVA caused a steric hindrance.

When analyzing functional groups using this method, it is important that only the functional group for the purpose is derivatized (the selectivity of the reaction). The result when derivatizing polymers have various functional groups is shown in Table 1. EVA and HEMA, which have hydroxyl groups, are derivatized. EBR (vinyl group), E-CO (carbonyl group), PAA (carboxyl group) and PMMA (ester group) are not derivatized. However, because N-epoxy (epoxy group) and Ny-6 (amide group) are derivatized, a measurement of hydroxyl groups in polymers which have epoxy or amide groups (e.g. epoxy resin, nylon) can not be performed using this method.

\section{Confirmation of the effectivity}

Hydroxyl groups are formed by an oxidation degradation of polymers, which has an alkyl chain, such as polyolefin, and also by a hydrolysis degradation of polyester. Therefore, hydroxyl groups are convenient for considering and evaluating of the oxidation degradation and the hydrolysis degradation of polymers. A neutralization titration method and a potentiometric titration method, among others, have been reported as methods for measuring the quantity of hydroxyl groups formed by the oxidation degradation and the hydrolysis degradation of polymers. These methods can measure the average of the functional groups in the polymer, but can not measure the distribution of the functional groups in the polymer. Therefore, by measuring the distribution of hydroxyl groups formed in actual polymer materials, the effectivity of this 

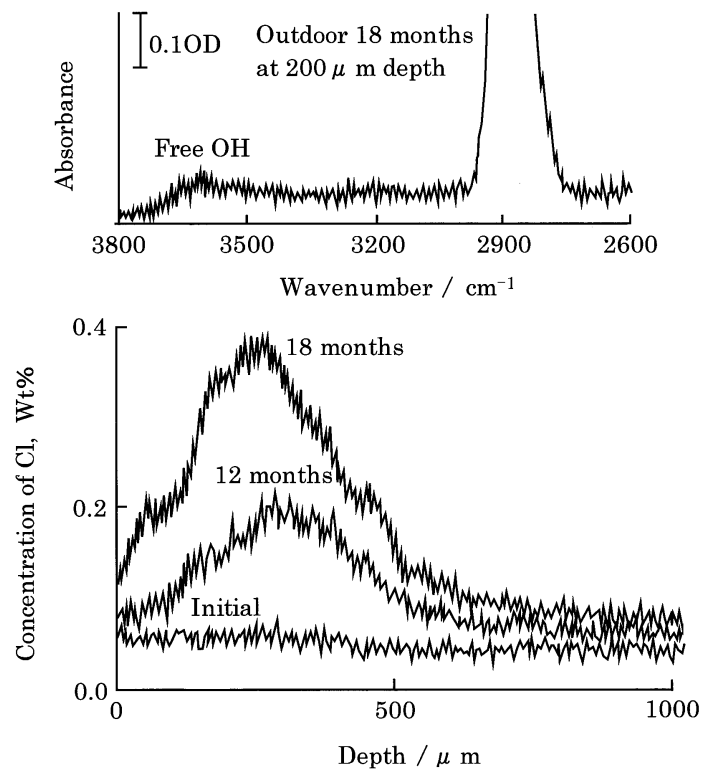

Fig. 5 Depth profiles of hydroxyl groups in polyethylene (PE) degraded by outdoor exposure (lower) and IR spectra at the peak top depth (upper).

method was confirmed.

1) Depth analysis of hydroxyl groups in outdoor-exposed polyethylene (PE). Polyethylene is a cheap and typical general plastic. Because its physical properties are excellent, it is widely used. Here, the depth profiles of hydroxyl groups in polyethylene (PE, $3 \mathrm{~mm}$-thickness plate, Sumitomo Chemical Co., Ltd) degraded by outdoor exposure were measured. Outdoor exposures were performed for 12 months and 18 months. The depth profiles of hydroxyl groups obtained by derivatization-XMA are shown in the lower section of Fig. 5. Also, a micro-IR spectrum at a position $200 \mu \mathrm{m}$ from the surface of outdoor-exposed PE for 18 months before derivatizing is shown in the upper section of Fig. 5 .

In PE exposed for 12 months and 18 months, hydroxyl groups which did not exist in the initial PE were generated from the surface to about $500 \mu \mathrm{m}$ depth. There was a high quantity of hydroxyl groups when the exposure period was long, but the quantity of hydroxyl groups near the surface was less than that inside. This phenomenon was thought to result from hydroxyl groups in the degraded PE being extracted by rain near the surface.

Because no absorption of hydroxyl groups was detected in the micro-IR spectrum of the exposed PE after 18 months, the high sensitivity of this method was confirmed. Also, when comparing the sensitivity of both methods from the $S / N$ of the hydroxyl group in the IR spectrum and the $S / N$ of the $\mathrm{Cl}$ element in the depth profile for 18 months, the sensitivity of this method was approximately 10-times that of micro-IR. Also, the detection limit $(S / N=2)$ of $\mathrm{Cl}$ elements in the polymer was $0.06 \%$ in a previous investigation. ${ }^{5}$ It became $0.03 \%$ when converting this detection limit to hydroxyl groups formed in PE. This sensitivity is equivalent to detecting about five hydroxyl groups among 10000 ethylene monomer units of PE.

2) Depth analysis of hydroxyl groups in acceleration-degraded polyester. Thermoplastic polyester is an engineering plastic that is widely used in bottles for drinking water, fibers, films and resins for formation, because its toughness, heat-stability,

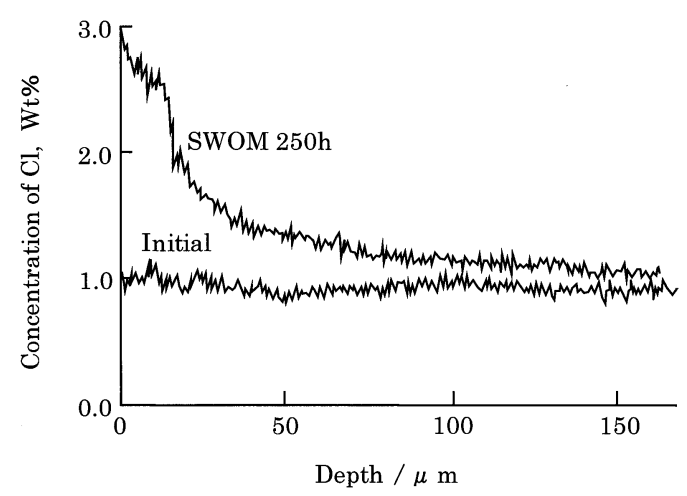

Fig. 6 Depth profiles of hydroxyl groups in polybutylene terephthalate (PBT) degraded by sunshine-weatherometer (SWOM).

chemical resistance and so on, are excellent. Here, the depth profiles of hydroxyl groups in polybutylene terephthalate (PBT, 3 mm-thickness plate, Toray Co., Ltd) degraded by the sunshine-weatherometer (SWOM) were measured. SWOM was implemented under the following conditions: irradiation times of $12 \mathrm{~min}$, water sprayer times of $48 \mathrm{~min}$, and total times of 250 h. The depth profiles of hydroxyl groups obtained by derivatization-XMA are shown in Fig. 6.

Although no hydroxyl groups were detected in the initial PBT, hydroxyl groups were detected near to the surface in PBT degraded for $250 \mathrm{~h}$. Under this condition, PBT degraded from the surface to approximately $80 \mu \mathrm{m}$ depth. We think that this degradation was caused mainly by the hydrolysis of the ester combination.

From the above examination, the distribution of $0.03 \%$ hydroxyl groups in the polymer could be measured by "derivatization-EPMA" that used trichloroacetic anhydride as the new derivatization reagent. Also, by applying this present method to a distribution analysis of hydroxyl groups in degraded polyethylene and polyester, the utility of this method in studying the degradation mechanism and in evaluating the degradation degree was confirmed.

\section{References}

1. M. A. Harthcock and S. C. Atkin, Appl. Spectrosc., 1988, $42,449$.

2. M. A. Harthcock, S. C. Atkin, and B. L. Davis, Microbeam Anal., 1988, 203.

3. T. Nishioka, T. Nakano, and N. Teramae, Appl. Spectrosc., 1992, 46, 1904.

4. A. Murase, Y. Esaki, M. Sugiura, and T. Araga, Anal. Sci., 1991, 7 (supplement), 1597.

5. M. Sugiura, A. Murase, T. Mitsuoka, and T. Araga, Bunseki Kagaku, 1994, 45, 251.

6. A. Murase, T. Mitsuoka, M. Sugiura, and T. Araga, Bunseki Kagaku, 1997, 46, 37.

7. M. Sugiura, M. Tsuji, A. Murase, and T. Mitsuoka, Bunseki Kagaku, 1999, 48, 983.

8. R. A. Dickie, J. W. Holubka, and J. S. Hammond, Org. Coatings Plast. Chem., 1979, 41, 499.

9. D. S. Evwehart and C. N. Reilley, Anal. Chem., 1981, 53, 665.

10. T. Ohmichi, H. Tamaki, and S. Tatsuta, Fuji photograph film study report, 1984, 29, 102. 